\title{
Stimulus-specific adaptation, habituation and change detection in the gaze control system
}

\author{
Yoram Gutfreund
}

Received: 22 January 2012 / Accepted: 23 May 2012 / Published online: 19 June 2012

(c) The Author(s) 2012. This article is published with open access at Springerlink.com

\begin{abstract}
This prospect article addresses the neurobiology of detecting and responding to changes or unexpected events. Change detection is an ongoing computational task performed by the brain as part of the broader process of saliency mapping and selection of the next target for attention. In the optic tectum (OT) of the barn owl, the probability of the stimulus has a dramatic influence on the neural response to that stimulus; rare or deviant stimuli induce stronger responses compared to common stimuli. This phenomenon, known as stimulus-specific adaptation, has recently attracted scientific interest because of its possible role in change detection. In the barn owl's OT, it may underlie the ability to orient specifically to unexpected events and is therefore opening new directions for research on the neurobiology of fundamental psychological phenomena such as habituation, attention, and surprise.
\end{abstract}

Keywords Optic tectum - Barn owl - Novely detection . Auditory · Superior colliculus · Adaptation .

Orienting response

\section{Habituation and saliency mapping}

Imagine a dog resting in the center of a noisy living room, easily ignoring the surrounding loud noises of kids and music playing but immediately raising its ears to the faint sound of the door knob turning. This describes a basic behavior in

This article is published as part of the Special Issue on Multimodal and Sensorimotor Bionics.

Y. Gutfreund ( $\square)$

The Department of Physiology and Biophysics, The Ruth \& Bruce Rappaport Faculty of Medicine, Technion, Haifa 31096, Israel

e-mail: yoramg@tx.technion.ac.il all animals. We have a remarkable capability of ignoring or habituating to the background noise, a trait that allows us to rapidly identify and respond to stimuli that break the regularity of the background (Thompson 2009). Note that such events need not necessarily be louder or brighter than the background; they must be different, or, in other words, unexpected. Thus, to some extent, animals are tuned to detect unexpected events or changes in the incoming streams of sensory information.

In nature, an unexpected event may predict danger or, for predators, an opportunity for food, therefore, a rapid response is fundamental for survival. Perhaps not surprisingly, mechanisms have evolved to respond selectively to unexpected events and to trigger a series of autonomic reflexes that prepare the body for possible action (Sokolov 1963; Barry 2009). These include galvanic responses (Bradley 2009), changes in heart rate (Bradley 2009), and pupillary dilation (Bala and Takahashi 2000; Weinberger et al. 1975). This wide repertoire of responses, which is remarkably preserved phylogenetically, has been termed by Pavlov as the "orienting response" (Sokolov 1963; Barry 2009). Orienting responses can include gaze shifts (overt orienting), but do not have to (covert orienting). In parallel to the orienting reflex, unexpected events also capture the animal's attention for evaluation and decision of the appropriate action (Posner 1980). Thus, unexpected events have dramatic and widespread bodily effects, most are hidden, pre-attentive, and subconscious, but can be detected with sensitive measurement devices (Bradley 2009).

The brain processing of unexpected events is part of a broader process called saliency mapping (Kayser et al. 2005; Itti and Koch 2000). A dog among cats, a brown object among orange objects or a pure tone succeeding a long period of broadband noise are all conspicuous stimuli that are perceptually salient. The first two examples are salient because they 
represent stimuli that are out of order in space; a brown object is found where an orange object is expected. Therefore, this type of saliency will be referred to here as spatial saliency. The last stimulus is salient because just like the sound of the door knob, it represents a stimulus that is out of order in time; a tone is heard during the time in which a broadband noise is expected, therefore this type of saliency will be referred to here as temporal saliency. Both temporal and spatial saliency are perceptually privileged; they give rise to attentional capture (Tiitinen et al. 1994; Posner 1980), enhanced autonomic responses (Weisbard and Graham 1971; Bala and Takahashi 2000), and enhanced neural responses (Ulanovsky et al. 2003; Reches and Gutfreund 2008; Naatanen 1995; Gottlieb et al. 1998). Given these similarities in the outcome, it is likely that the neural mechanisms for mapping spatial saliency overlap with the neural mechanisms for mapping temporal saliency. In summary, the basic task of saliency mapping links together fundamental psychological phenomena such as attention, habituation, orienting, and surprise, phenomena that are in many cases studied separately.

This prospect will focus on temporal saliency. Mapping the temporal saliency of an incoming stimulus is an ongoing task that involves some of the basic building blocks of neural computation. It involves creating an expected template or model of the world based on the past, memorizing the template, comparing the incoming input with the expected template, detecting violations, and finally updating the expected template. Therefore, this task is of general interest not only to experimentalists but also to computational neuroscientists interested in modeling the brain (Mill et al. 2011). In the following sections, I will address the question of where and how unexpected events are computed and represented in the brain, and discuss the relationships between temporal saliency mapping, habituation, and attention.

\section{Neural correlates of temporal saliency mapping}

\subsection{Mismatch negativity}

Neural correlates of temporal saliency mapping or change detection have been exposed and studied in humans using scalp electroencephalogram recordings, mostly in the auditory system. The auditory event-related potential (ERP) contains several components, one of which is a negative deflection, peaking between 100 and $200 \mathrm{~ms}$ after stimulus onset. This negative component is called N1. N1 tends to decrease with repeated stimulation reflecting the adaptation of neural responses (Nelken 2004). However, the neural response not only adapts but also predicts the upcoming stimulus based on the past. This we know because when a sound violates the regularity of the repetition (a deviant sound), a new ERP component is recorded called mismatch nega- tivity (MMN; Naatanen 1995). MMN generators have been linked to the auditory cortex (Alho 1995) and are clearly preattentive because they can be measured in humans engaged in a completely non-auditory task such as reading a book (Sams et al. 1985). Moreover, MMN can be measured independent of subject awareness to the deviant sounds or even in some cases in unconscious subjects (Kane et al. 1993). MMN can be elicited by almost any detectable deviation, independent of the specific feature. Rare changes in stimulus frequency, intensity, duration, spatial location, or irregularities in spectrotemporal sequences can all elicit MMN (Naatanen 1995; Naatanen et al. 2001). Moreover, significant MMN is recorded in cases where the difference between the deviant and the standard is minute, comparable to the minimal noticeable difference (Nelken and Ulanovsky 2007). Thus, the study of MMN demonstrates that neural representation of unexpected events in the human brain is robust and highly sensitive. This should not come as a surprise given the importance of unexpected events in animal behavior (discussed above). However, because ERPs reflect a global, indirect signal, their study is limited in its ability to reveal fine details of the neural circuits that compute and represent deviations. For this purpose, identifying neural correlates of temporal saliency mapping at the single unit level is highly important.

\subsection{Stimulus-specific adaptation}

Neural adaptation, the decline in response during prolonged or repetitive stimulation, is a ubiquitous property of sensory neurons. Two major types of adaptations have been described in the literature: one is the non-specific adaptation that depends on the activation history of the neuron more than on specific features of the stimulus (Calford and Semple 1995; Brosch and Schreiner 1997; McAlpine et al. 2000; Ingham and McAlpine 2004; Furukawa et al. 2005; Wehr and Zador 2005; Gutfreund and Knudsen 2006); the other, a higher level adaptation, is stimulus-specific adaptation (SSA), an adaptation to the history of the stimulus rather than to the activity of the neuron (Ulanovsky et al. 2003, 2004). To distinguish SSA from non-specific adaptation, several stimulation paradigms have been used (Fig. 1). In all paradigms, the responses to standard, common stimuli are compared with responses to the same stimuli, but when presented rarely or as deviants. SSA implies that the neuron responds more strongly to stimuli when deviant (see illustrations in Fig. 1). Therefore, this phenomenon has been suggested as a single-unit neural correlate of the detection of unexpected stimuli (i.e., change detection, sometimes known as novelty detection; Ulanovsky et al. 2003; Perez-Gonzalez et al. 2005; Malmierca et al. 2009).

Using the above-mentioned criterion, it was shown that SSA is a common phenomenon in the brain and has been observed in visual (Muller et al. 1999), somatosensory (Katz 
A Priming

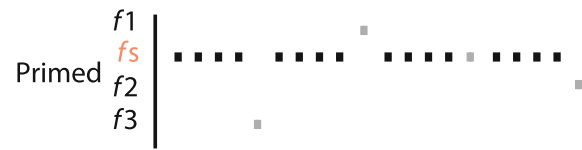

$f 1$
Control $f s$
$f 2$
$f 3$

Non-specific adaptation

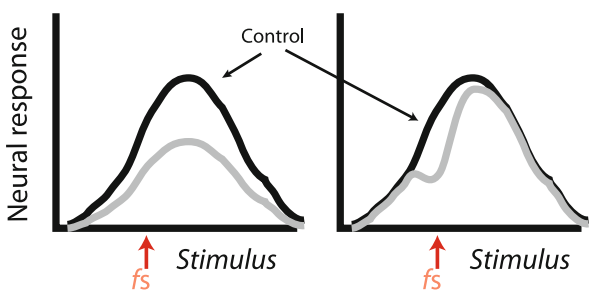

B Oddball
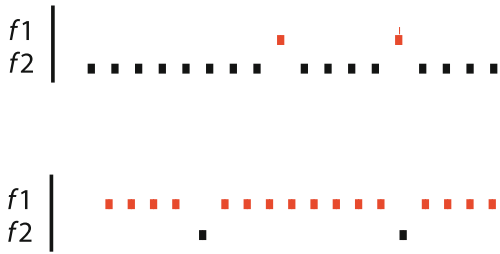

Non-specific adaptation

SSA

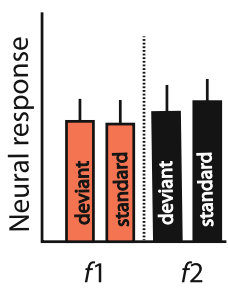

C Repeated sequence
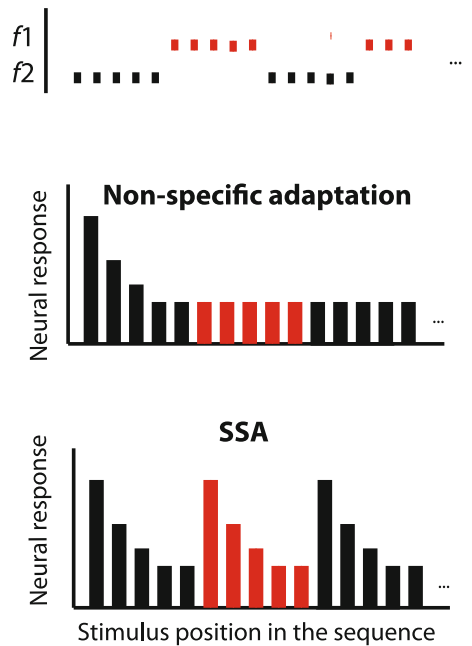

Fig. 1 Schematic representation of stimulus protocols used to differentiate SSA from non-specific adaptation. a The priming protocol. In the priming protocol, a tuning curve is generated by presenting sequences of stimuli varying randomly over a range of stimulus values $(f 1-f n)$. This is repeated under two conditions: in one (the control), the stimuli for generating the tuning curve are spaced with a fixed time interval of no stimulation; in the other (the primed), a standard stimulus $(f s)$ is presented several times in between stimuli. In non-specific adaptation (left graph), the tuning curve in the primed condition (light curve) is expected to be evenly attenuated relative to the control (black curve). In SSA, the adaptation is expected to occur stronger near the $f s$ frequency, resulting in a dip in the tuning curve. $\mathbf{b}$ The oddball protocol. In the oddball protocol, different stimuli are chosen ( $f 1$ and $f 2)$ so that both are within the response range of the neuron, giving rise to about the same response. One stimulus is chosen arbitrarily as the standard, being presented repeatedly for a long period. Within this sequence, the second stimulus called the deviant is embedded with a relatively low probabil-

et al. 2006), and auditory (Perez-Gonzalez et al. 2005) pathways. In the auditory system, neurons sensitive to deviations were found at different levels of the pathway: in the inferior colliculus, the auditory thalamus and the auditory cortex (Malmierca et al. 2009; Anderson et al. 2009; Farley et al. 2010; Antunes et al. 2010; Ulanovsky et al. 2004). Detailed characterization of SSA in the auditory system revealed that it is highly sensitive to minute deviations from the standard frequency. Neurons have been found to respond significantly more strongly to stimuli that are deviant from the standard by a frequency difference $<$ as small as $0.1 \%$ (Ulanovsky et al. 2003). Moreover, it was shown that this adaptation has several time scales ranging from sub-seconds to tens of seconds (Ulanovsky et al. 2004). This implies that the expected model can be updated on a rapid time scale to encompass rapid changes in the stimulus environment, but at the same time a longer history of stimulation is allowed to affect subsequent responses. In summary, recent studies on auditory ity. Later, a second block is presented in which the roles are changed: the stimulus that was standard is now deviant, and vice versa. At the end of the experiments, the average responses to the standards are compared with the corresponding responses to the deviants. In non-specific adaptation, we expect no significance differences (left histogram). In SSA, we expect the responses to the deviants to appear consistently above the responses to the standards (right histogram). $\mathbf{c}$ The repeated sequence protocol. In the repeated sequence protocol, the two stimuli are presented in a long sequence starting with several repetitions of $f 1$ then switching to several repetitions of $f 2$, and so on, alternating between the two stimuli. In non-specific adaptation, we expect that the response to the first stimulus in the sequence will be larger, further stimulation will result in adaptation that is independent of the type of stimulus (upper histogram in c). In SSA, we expect a stronger response every time the sequence is switched from one stimulus to the other (lower histogram in $(\mathbf{c}))$

SSA in the cortex, the thalamus, and the inferior colliculus suggest that single-neuron sensitivity to changes in the input stream is robust and widespread. The origin and mechanisms of this phenomenon is yet to be discovered. An additional open question is the role of this type of SSA in perception and behavior. Three main problems discussed below hinder the attempts to relate this neural phenomenon to the psychological phenomena of habituation and saliency mapping.

\subsubsection{The multiple feature problem}

SSA in the auditory cortex and the inferior colliculus has been studied mostly using pure tone stimuli where the deviants differed from the standards by the frequency. Other sensory features were either not studied or gave rise to poor SSA (Farley et al. 2010; Ulanovsky et al. 2003). In nature, a stimulus can differ from what has been in the past along multiple features, i.e., frequency, amplitude, duration, location, etc., 
or combinations of features. A neuron that is sensitive to changes in the frequency of the stimulus but not to changes in other features is a limited "change detector," which cannot explain the high sensitivity to deviant stimuli independent of the stimulus feature observed behaviorally and at the EEG level. It is therefore necessary to identify types of SSA that encompass multiple sensory features.

\subsubsection{The memory trace problem}

The memory trace of adaptation is the time it takes from the last stimulus until its effect on the response to the next stimulus wears out. In the laboratory, this is measured by presenting sequences of stimuli with various inter-stimulus intervals (ISIs). The memory trace is the minimal ISI in which the response to a stimulus is not affected by it's preceding stimulus. SSA in the auditory cortex or the thalamus has been reported to diminish at ISIs longer than $2 \mathrm{~s}$ (Ulanovsky et al. 2003) or has only been studied at ISIs $<2$ s (von der Behrens et al. 2009; Antunes et al. 2010). Therefore, we can conclude that the information about the standard is stored in memory for only about $2 \mathrm{~s}$. This poses a major problem because many examples of behavioral habituation have been reported with ISIs of tens of seconds to minutes, even for short duration stimuli (Thompson and Spencer 1966; Weinberger et al. 1975; Bala and Takahashi 2000; Zimmer 2006; Glanzman 2009; Dong and Clayton 2006; Valentinuzzi and Ferrari 1997). It is therefore necessary to identify a form of SSA that maintains a longer memory trace.

\subsubsection{Change detection versus probability detection}

Most studies of SSA in the auditory pathways were conducted using standard oddball paradigms, whereby a deviant frequency is embedded with a certain probability in the sequence of standard frequency (Fig. 1b). In such a probabilistic stimulus, SSA implies that the response of the neuron is modulated by the probability of the deviant; the smaller the probability, the larger the response. However, sensitivity to probability is not necessarily equal to temporal saliency mapping. The latter requires learning an expectation rule set by the standard and pointing out any deviations from this rule, while the former requires simply counting the number of stimuli over a period of time and responding accordingly. Rare stimuli are not always salient. For example, a stimulus can appear in a sequence of multiple different stimuli, each being rarely presented but none is salient from the others (Taaseh et al. 2011). The standard oddball paradigm cannot distinguish between the two possibilities. Despite recent attempts to resolve this issue for SSA in the auditory cortex, it still remains an open question (Furukawa et al. 2005; Taaseh et al. 2011).

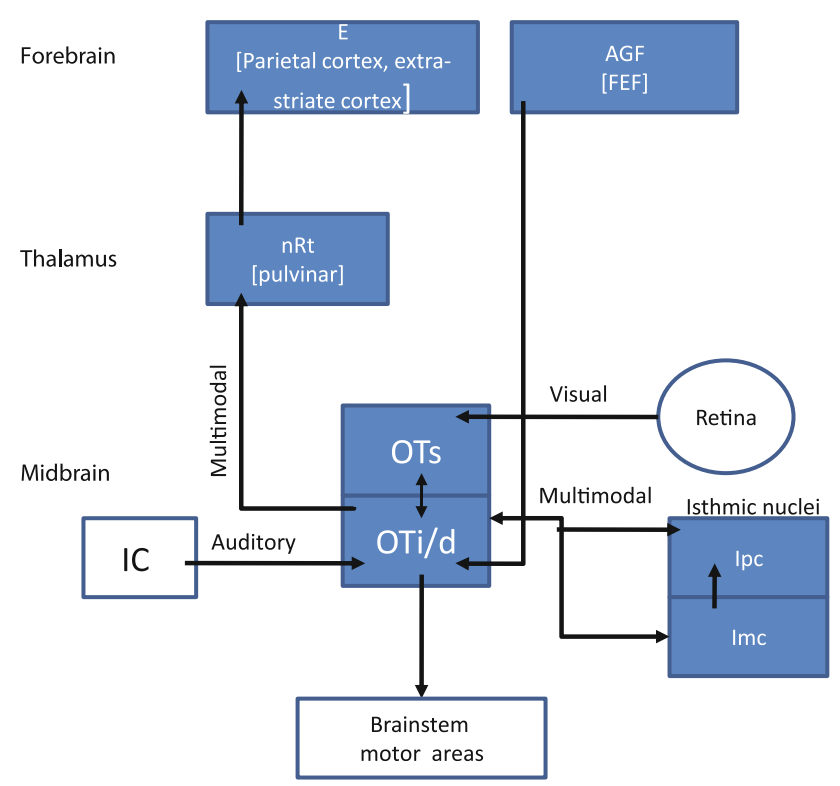

Fig. 2 An illustration showing a selected part of the avian gaze control circuitry with the OT as a hub. Filled boxes indicate structures suggested to be related to gaze and attention control. Double-head arrows indicate reciprocal connections. The names of suggested equivalent mammalian brain areas are pointed out in the square brackets. $E$ entopallium, $A G F$ arcopallium gaze fields, $F E F$ frontal eye fields, $n R t$ nucleus rotundus, OTs superficial layers of the OT (layers 1-10), OTi/s intermediate and deep layers of the OT (layers 11-15), IC inferior colliculus, Ipc nucleus isthmi pars parvocellularis, $\operatorname{Imc}$ nucleus isthmi pars magnocellularis

\section{Gaze control and saliency mapping}

All vertebrates possess a specialized brain system responsible for orienting the body toward stimuli of interest. This system, known as the gaze control system (sometimes called the oculomotor system), contains a number of mid-brain and forebrain areas (see scheme of basic circuitry in Fig. 2). The superior colliculus (SC), arguably one of the most phylogenetically conservative structures in the brain (Gaither and Stein 1979; Shimizu and Karten 1993; Luksch 2003), is a mid-brain structure that is a critical hub in this system (Boehnke and Munoz 2008). The avian homolog of the $\mathrm{SC}$ is the optic tectum (OT). Both mammalian and avian structures are laminated and demonstrate many similarities in their input and output patterns (Shimizu and Karten 1993; Knudsen 2011). Pre-motor neurons in the OT/SC send projections to motor nuclei (Masino and Knudsen 1992; Rodgers et al. 2006), and microstimulation in the OT/SC readily induces eye and head shifts in a wide variety of species (McHaffie and Stein 1982,; Sparks 1986; Herrero et al. 1998; Netser et al. 2010). Hence, the OT/SC is primarily known as a gaze control center serving to control eye and head movements; however, this textbook description of the OT/SC is reducing the full breadth of its functional role. Mounting evidence shows that the OT/SC contains more than a simple motor system. Information from the OT/SC is also sent to 
a wide range of cortical and basal ganglia regions via thalamic nuclei (Robinson and Petersen 1992; Takada et al. 1985; Bischof and Watanabe 1997; Reches and Gutfreund 2009). An emerging hypothesis is that the evolutionary role of the OT/SC is to sort stimuli based on saliency, select the most salient stimulus, and send this information to the appropriate brain regions to direct orienting movements, attention, and autonomic responses (Review in Boehnke and Munoz 2008; Knudsen 2011).

The above-mentioned hypothesis is supported by numerous electrophysiological studies demonstrating that neurons in the OT/SC are sensitive to the saliency of the stimulus inside their receptive field (for example: Horwitz and Newsome 1999; McPeek and Keller 2002; Pluta et al. 2011). Further evidence for the causal role of the SC in stimulus selection and spatial attention was provided by several inactivation and microstimulation studies (Muller et al. 2005; Lovejoy and Krauzlis 2009). Of particular interest to this prospect is a series of recent studies in barn owls addressing mechanisms of competitive stimulus selection in the OT (Mysore and Knudsen 2011; Mysore et al. 2011, 2010; Lai et al. 2011). It has been suggested that competitive interactions are mediated by a GABAergic midbrain nucleus (Imc, nucleus isthmi pars magnocellularis), which receives topographic connections from the OT directly and indirectly through a nearby cholinergic nucleus (reviewed in Mysore et al. 2011). However, all the studies cited above used experimental settings which highlight spatial saliency, i.e., the selection of a stimulus among simultaneously presented stimuli. As discussed above, an important element in stimulus saliency is its history. Stimuli that differ from previous stimuli (surprising stimuli) are perceived as being more salient (Posner 1981; Itti and Koch 2000). If saliency mapping is fully performed in the OT, we would expect that temporal saliency will also be represented in the responses of tectal neurons. The role of the OT/SC in temporal saliency mapping or change detection has not been studied as much as spatial saliency. It is an open question as to what extent the neural circuits that highlight a salient stimulus in space overlap with the circuits that highlight a salient stimulus in time. Below I will discuss this question in light of recent data from the barn owl's OT.

\section{The barn owl's OT}

The deep and intermediate layers of the barn owl's OT receive topographic auditory inputs from the inferior colliculus (Knudsen and Knudsen 1983; Feldman and Knudsen 1997; Hyde and Knudsen 2000) (Fig. 2). Neurons in these layers respond to sounds that arrive from a certain location in space (space-specific neurons) and are organized to form a mapped representation of auditory space
(Knudsen and Konishi 1978; Knudsen 1982). An auditory map is not unique to barn owls; all species studied show some degree of topographic representation of auditory space in the OT (Lewald and Dorrscheidt 1998; King and Palmer 1985; King and Hutchings 1987). However, this map in the barn owl is probably the most organized and has the highest resolution of all (Knudsen 1987). Auditory space-specific neurons are derived from the integration of a variety of localization cues that arise from the interaction of incoming sound with the physical properties of the head and ears (Middlebrooks and Green 1991). In the owl's brainstem, two independent pathways process the binaural spatial cues (Moiseff and Konishi 1983; Takahashi et al. 1984): interaural time difference (ITD), which results from a difference in the distance traveled by a sound to reach the left versus the right ear; and interaural level difference (ILD) which arises from the frequency-dependent directional properties of the external ears. In barn owls, ITD is the main cue for designating the horizontal position of the sound and ILD for designating the vertical position of the sound (Moiseff 1989; Poganiatz et al. 2001; Poganiatz and Wagner 2001).

The superficial layers of the OT receive topographic visual inputs directly from the retina (Fig. 2), as well as indirectly from visual forebrain areas (Karten et al. 1973), giving rise to a retinotopic representation of visual space (Knudsen 1982). Since the superficial and deep layers are functionally connected, many neurons in the OT are multisensory, responding to both visual and auditory signals inside their receptive fields (Brainard and Knudsen 1998; Zahar et al. 2009).

\subsection{Stimulus-specific adaptation in the OT}

Numerous studies have reported that sensory neurons in the SC/OT tend to adapt to repeated visual or auditory stimulation (Woods and Frost 1977; Fecteau and Munoz 2005). Recently, auditory adaptation was characterized systematically in the barn owl's OT (Reches and Gutfreund 2008; Netser et al. 2011). Oddball and repeated sequence protocols (see Fig. $1 \mathrm{~b}$ and c, respectively) were used to demonstrate that adaptation in the OT is, to a large extent, stimulus specific. Most neurons in the OT responded stronger to a low probability stimulus or to a stimulus that was different from its past. For example, Fig. 3 a shows the average response of multiple neurons recorded in the OT as a function of the position of the stimulus in a sequence. In this case, the stimulation paradigm was cyclical, alternating every 10 stimuli between two ITD values (see Fig. 1c). It can be seen that every time a switch occurred between one ITD to another, the response increased and adapted again until the next switch occurred. Remarkably, significant changes in neural responses were observed even when the stimulus was deviant by an ITD as small as $20 \mu$ s (Reches and Gutfreund 2008). Thus, the neural response to an auditory stimulus depended not only on the 
- |||||||||||||||||||||||||||| $\mid \cdots$ $\stackrel{\longrightarrow}{\text { Time }}$

A

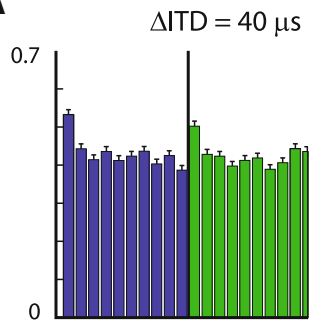

C

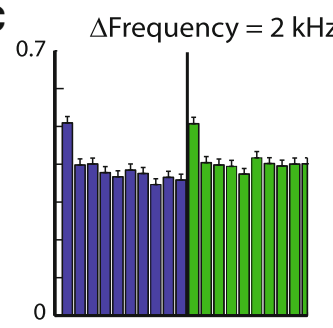

E

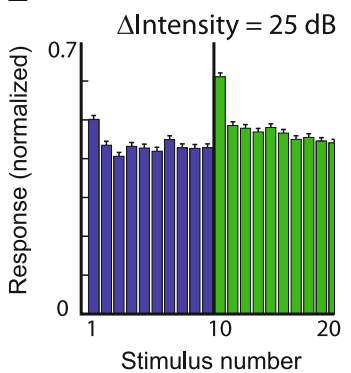

B

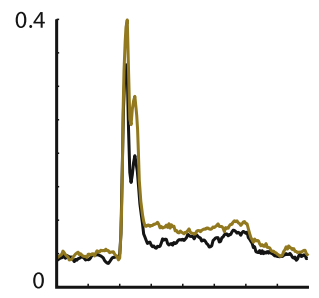

D

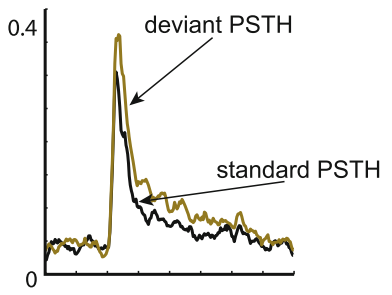

$\mathbf{F}$

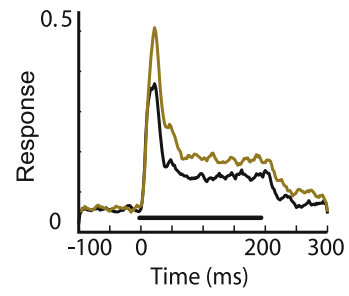

Fig. 3 Stimulus-specific adaptation in the OT of the barn owl to multiple acoustic features. a The histogram shows the average response of a population of neurons in the OT to a long sequence of stimuli (200 stimuli). The duration of each stimulus was $200 \mathrm{~ms}$ and the ISI was $1 \mathrm{~s}$. The sequence alternated between two ITD values; every 10 repetitions, the ITD of the sound was switched to the other value (sequence is illustrated in inset). b The post-stimulus time histogram (PSTH) of one stimulus when it was first in its sub-sequence (deviant, light red curve) is compared to the PSTH for the same stimulus when it was last in its sub-sequence (standard, black curve). c, d Same as a and b, but in this case, the two stimuli were narrowband stimuli (width of $1 \mathrm{kHz}$ ) differing in central frequency. e, f Same as a and $\mathbf{b}$, but in this case, the two stimuli differed in intensity. One stimulus (dark blue bars) was softer compared to the other (light red bars). The average response to deviant ITDs was greater in all cases. Modified with permission from Reches and Gutfreund (2008). (Color figure online)

value of the ITD of the stimulus but also on how this value differed from previous ITD values.

Sensitivity to changes in the input stream was not limited to changes in the ITD of the sound. Testing for changes in the frequency, the intensity, or the ILD of the sound gave rise to the same basic result, i.e., the neurons readily detected changes in the input stream along all stimulus dimensions (Reches and Gutfreund 2008). In all cases (ITD, ILD, intensity, and frequency), the adaptation was stimulus specific and developed rapidly after one trial (Fig. 3). This similarity is especially striking taking into account that the four features

are represented and computed in markedly different ways: ITD and ILD, the two primary binaural localization cues, are processed in parallel in two separate and independent brainstem pathways (Takahashi et al. 1984; Takahashi and Konishi 1988; Adolphs 1993; Albeck and Konishi 1995); frequency separation is maintained in both ascending auditory pathways from the cochlea up to the level of the lateral shell of the inferior colliculus where information across frequency-specific channels is combined (Euston and Takahashi 2002). Sound-level information is presumably manifested in the response levels of the ascending pathways. Therefore, the fact that all four independent acoustic features showed a qualitatively similar pattern of adaptation suggests that SSA is an important property in the neural representation of the auditory scene in the OT. Possibly, this property underlies the owl's ability to attend and orient abruptly to novel events.

The robustness of SSA in tectal neurons gives rise to an interesting ambiguity problem, i.e., an inability to discriminate between two conditions. For example, a soft sound usually produces weaker responses in the OT compared to a louder sound; however, the same soft sound can induce stronger neural responses than the loud sound when it is deviant in an environment characterized by loud sounds (see Fig. $3 e, f)$. Thus, neural responses in the tectum seem unable to code unambiguously the sensory identity of the incoming sound. This, together with the finding that OT/SC neurons are mostly broadly tuned to sensory features, such as frequency, amplitude modulations, orientation, direction, and modality, (Mize and Murphy 1976; Zahar et al. 2009) is consistent with the hypothesis that indicating the exact identity of the stimulus is not a computational task of the OT. The OT represents the location of the stimulus and how salient it is in time and space. Stimulus identity is presumably processed in a different pathway.

\subsection{Interactions between multisensory integration and SSA}

An additional finding is that tectal neurons are also sensitive to deviants in the visual input stream (i.e., visual SSA; Woods and Frost 1977; Reches and Gutfreund 2008), and more importantly, SSA in the visual modality can be modulated by the co-occurrence of auditory stimuli (Reches et al. 2010). The experiments showing this were performed in a forebrain area called the entopallium (E). In barn owls, this region receives visual and auditory inputs originating in the OT (Fig. 2; Reches and Gutfreund 2009). Nevertheless, its neurons are far more sensitive to visual stimuli compared to auditory stimuli. Figure 4 shows the average response histograms of a cluster of neurons (multi-unit site) in entopallium to a typical oddball test. The neurons demonstrated clear visual SSA; they responded much stronger to the deviant visual stimuli compared to the same stimuli when common (Fig. 4d, e). These same neurons did not respond to 
A
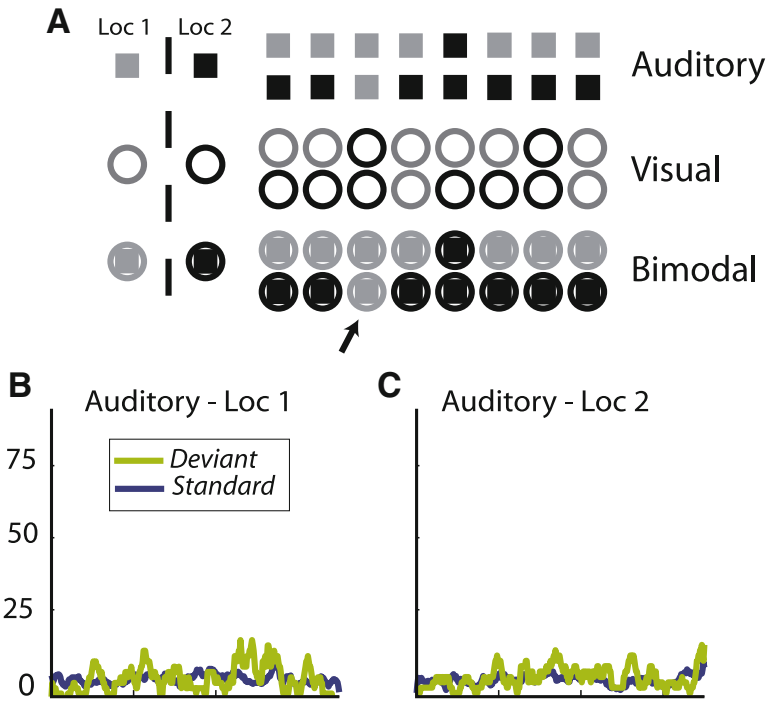

C Auditory - Loc 2
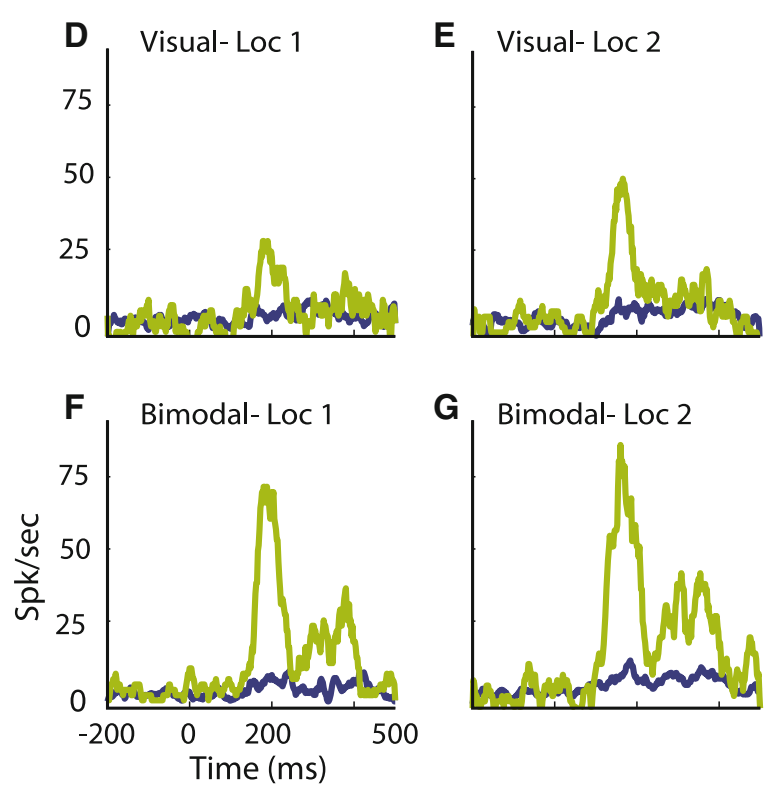

Fig. 4 Visual SSA is modulated by auditory stimuli. a Outline of the bimodal oddball test. Each line represents a sample sequence from a single oddball block. Squares designate the auditory stimuli, circles the visual stimuli and enclosed squares the bimodal stimuli. Gray shaded shapes specify stimuli from one location (Loc 1) and black shapes specify stimuli from the second location (Loc 2). The two locations were spaced horizontally by $20^{\circ}$. The arrow indicates an example of a deviant bimodal stimulus. b-g PSTHs of responses to deviants (light red lines) are shown together with the corresponding responses to standard presentations (dark blue lines). Responses to the two auditory stimuli are shown in $(\mathbf{b}, \mathbf{c})$, the two visual stimuli in $(\mathbf{d}, \mathbf{e})$ and the two bimodal stimuli in (f, g). Modified with permission from Reches et al. (2010). (Color figure online)

the auditory stimuli no matter if the stimulus was deviant or standard (Fig. 4b, c). Interestingly, when both the auditory and the visual stimuli were presented simultaneously from the same deviant location (arrow in Fig. 4a), the response to the bimodal stimulus was enhanced dramatically, whereas very little enhancement occurred when the visual and auditory stimuli were presented simultaneously from a standard location (Fig. 4f, g). Thus, it seems that obtaining congruent information from different senses may improve the sensitivity of the neurons to deviations in the input stream. Saliency mapping is usually studied in unimodal settings; however, in nature it is primarily an amodal task. The saliency of an event is determined by a combination of modalities (Stein and Meredith 1993; Pluta et al. 2011) and therefore we expect multisensory integration to take place in the saliency mapping pathways.

\subsection{The memory trace of adaptation}

A common notion in the adaptation of neural responses, backed up by computational models of synaptic suppression (Tsodyks and Markram 1997), is that the dynamics of neural adaptation complies with stimulus duration (Marom 2009): a short duration stimulus is expected to induce short-lasting adaptation, and vice versa (Varela et al. 1997; Ulanovsky et al. 2004). This concept is in line with most studies of auditory SSA described above including those in the barn owl. In these studies, the ISIs varied between $300 \mathrm{~ms}$ and $2 \mathrm{~s}$, stimulus durations were in the range of $100-500 \mathrm{~ms}$, and the probability of the deviant was $10-15 \%$. None of the above cited papers reported a memory trace longer than $2 \mathrm{~s}$, which is within the time scale of the stimulus timing. However, as mentioned earlier, this relatively short memory trace constitutes a major problem for linking neural SSA with mechanisms of habituation. Behavioral habituation does not comply with the above-mentioned principle of comparable time scales. For example, in the barn owl, reflexive pupil dilation and eye movements to sequences of relatively short stimuli with ISIs of 10-13 s readily habituated and recovered when the stimulus was switched from one type to another (Bala and Takahashi 2000; Spitzer et al. 2003; Netser et al. 2011).

To close the time gap between behavioral habituation and SSA in the OT, it is important to examine the memory trace of the SSA. This was done in the OT of barn owls by presenting sequences of identical sounds and measuring unit responses as a function of the position of the stimulus in the sequence. Remarkably, it was found that the memory trace of the SSA in the OT can be up to $60 \mathrm{~s}$ long (Netser et al. 2011). A single, short $(300 \mathrm{~ms})$, and low intensity ( $20 \mathrm{~dB}$ above threshold) stimulus was sufficient to induce a reduction in the neural response to the same stimulus occurring $60 \mathrm{~s}$ later. Moreover, the system not only memorized that there was a stimulus $60 \mathrm{~s}$ earlier but also what type of stimulus because presenting a third stimulus different from the first two, after an additional $60 \mathrm{~s}$, resulted in complete recovery of the response (Fig. 5c, d). The finding that specific adaptation in the OT can reach time spans of over a minute suggests that this type of neural adaptation is a neural correlate of 

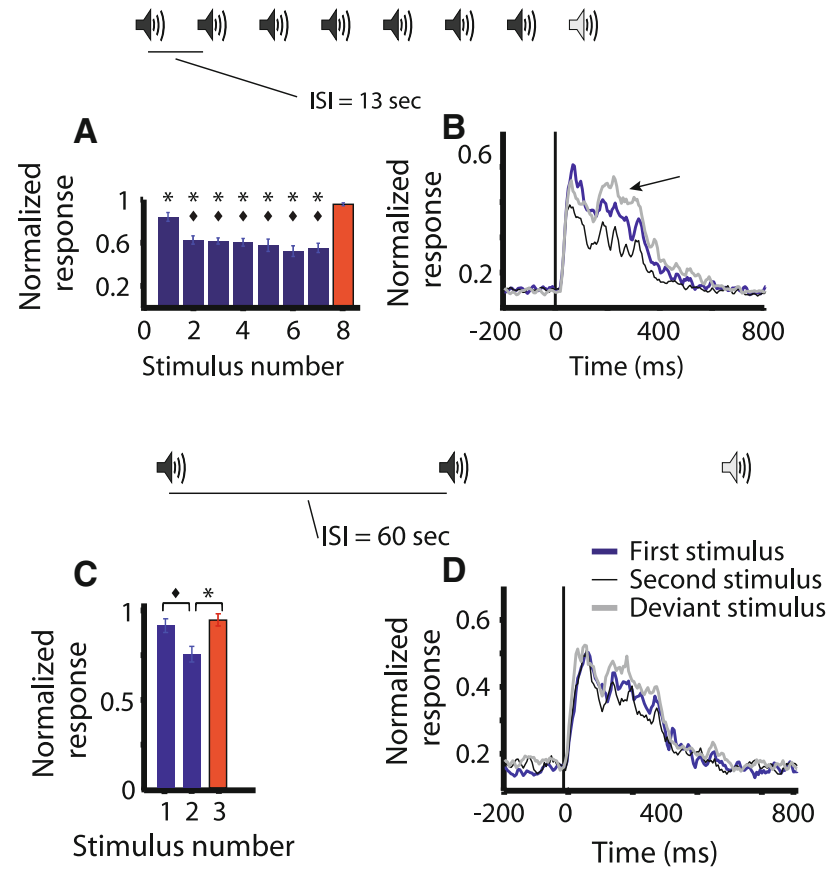

Fig. 5 SSA with relatively long ISIs in the OT. a The average response of a population of neurons in the OT as a function of the position of the stimulus in a sequence with an ISI of $13 \mathrm{~s}$. The sequence was composed of seven repetitions of the standard sound followed by one presentation of an odd sound (see inset). The dark blue bars designate responses to the seven repeated presentations of the standard stimulus and the light red bar designates the response to the last odd stimulus. Error bars indicate SEMs. Diamonds indicate responses that were significantly smaller than the response to the first stimulus. Asterisks indicate responses that were significantly smaller than the response to the last odd stimulus. b The average PSTH curve of the response to the first stimulus (thick dark curve) compared to the average PSTH curve of the second stimulus (thin dark curve) and the average PSTH curve of the response to the last odd stimulus (gray curve). The arrow points to the region where the response to the odd stimulus is facilitated. $\mathbf{c}$ The average response of a population of neurons in the OT as a function of the position of the stimulus in a sequence with an ISI of $60 \mathrm{~s}$. The sequence was composed of two repetitions of the standard sound followed by one presentation of an odd sound (see inset). The dark blue bars designate the responses to the two repeated presentations of the standard stimulus and the light red bar designates the response to the last odd stimulus [format as in (a)]. d The average PSTH curve of the response to the first stimulus (thick dark curve) compared to the average PSTH curve of the second stimulus (thin dark curve) and the average PSTH curve of the response to the last odd stimulus (gray curve). Modified with permission from Netser et al. (2011). (Color figure online)

behavioral habituation. Further supporting the link between tectal neural circuitry and habituation of the orienting reflex is the recent finding that low current microstimulation in the OT just before an auditory stimulus resulted in an increase in an already habituated eye shift response to the auditory stimulus, i.e., activation of tectal circuitry can induce recovery from habituation to a certain extent (Netser et al. 2010).

Close inspection of the average responses in Fig. 5b and $\mathrm{d}$ reveals a noteworthy observation. The responses to the deviant stimulus were on average greater than the responses to the same stimulus when it was first in the sequence (arrow in Fig. 5b). Thus, the change from a standard stimulus pattern to a new stimulus elicited stronger responses than the initiation from silence of a new stimulus. Such a result, which enhances change detection, cannot be explained by simple adaptation models, some facilitation is involved in the process. Interestingly, no such facilitation was observed when the ISIs were smaller than $2 \mathrm{sec}$. In the short ISIs, the responses to the deviant stimulus were usually smaller than the responses to the same stimulus when presented alone (Reches and Gutfreund 2008a; Taaseh et al. 2011). The facilitation of the responses to deviant stimuli occurred at late stages of the response, 150-200 ms after the onset of the stimulus. This is reminiscent of the MMN signal in humans (see above) and suggests that the computation of change detection occurs at high levels of the pathway.

\section{Final remarks and open questions}

SSA is a neuronal phenomenon that resembles change detection and habituation in many aspects. However, not all types of SSA can serve as a neural correlate of habituation. In this prospect I have pointed out a few requirements, namely the ability to detect changes in multiple features and modalities, the ability to maintain a relatively long memory trace, and the ability to detect salient changes, i.e., stimuli that break the regular pattern and not just rare stimuli. We argue that most types of SSA reported so far do not fulfill these requirements. Recently, however, a new type of auditory SSA was reported in the OT of the barn owl that is sensitive to multiple features and modalities, maintains a relatively long memory trace that is correlated with behavior, and involves facilitation of responses to sounds that deviate from the background. These findings suggest that this type of SSA is a neural correlate of habituation and temporal saliency mapping. Together with many other studies, the findings point to the OT and its related circuitry as a hub for both temporal and spatial saliency mapping, and are opening new directions for research on the mechanisms of habituation and attention control. Several open questions regarding these new findings are discussed below.

\subsection{What are the neural mechanisms of SSA?}

The neural mechanisms underlying SSA in the auditory pathways as well as in the OT are unknown. One common model to explain SSA is that the two stimuli activate separate paths to the recorded neuron and that basic adaptation mechanisms (synaptic depression or intrinsic cellular mechanisms) act at levels where the activation is separated (Eytan et al. 2003). An intriguing observation in the barn owl's SSA is a complete 
lack of cross-stimulus adaptation at long ISIs even though the frequency content of the two stimuli overlapped substantially (Netser et al. 2011). It is therefore unlikely that this type of SSA is accounted for solely by basic response suppressions at lower, frequency-specific levels. To compute the novelty of complex broadband sounds, a network is required which compares the neural responses to the current stimulus with previous responses based on an integration of information about frequency and amplitude modulations. The details of such a network are yet to be discovered.

\subsection{What is the relationship between SSA and behavior?}

The phenomenon of SSA has been studied mostly in anesthetized animals. What effects it has on behavior is yet to be shown. As mentioned earlier, not all types of SSA are necessarily linked to behavioral habituation and saliency mapping. Some may be related to scene analysis or optimal coding (reviewed in Winkler et al. 2009). One approach for studying the relationships between neural adaptation and behavior would be to record simultaneously behavioral and neuronal responses and examine the trial-by-trial correlations between SSA and behavioral habituation. Another approach would be to inactivate brain areas which contribute to SSA and examine the effects on the behavioral habituation and on the animal's ability to respond to changes in the environment.

\subsection{How and where is saliency mapping modulated by internal priorities?}

So far, we have discussed saliency mapping as a purely bottom-up process. Within this framework, the saliency of an object or an event is based on external factors alone (stimulus intensity, stimulus history, spatial context, cross-modal interactions, etc.). However, behavioral selection of a stimulus is guided by a combination of external saliency and internal factors (cognitive biases, behavioral tasks, reward history, motivations, etc.). For example, when one is searching for a red book, red objects become salient. Therefore, somewhere in the brain such "top-down" information about the relevance of the event to the animal must be integrated with information about the "bottom-up" saliency of the event. Where in the brain does this integration happen and what are the mechanisms involved is an open question. One hypothesis supported by several experimental results (reviewed in Boehnke and Munoz 2008; Knudsen 2007) is that neurons in the SC integrate "bottom-up" with "top-down" information to signal the most important stimulus at any particular time. It is suggested that task-related information about the stimulus is conveyed to the SC through cortical and basal ganglia connections (Boehnke and Munoz 2008). In the barn owl, forebrain-tectal connections have been shown to modulate visual and auditory tectal responses in a space-specific manner (Winkowski and Knudsen 2006, 2007). These modulations are conveyed by the arcopallium gaze field (AGF), a forebrain area suggested as an equivalent to the frontal eye fields (Knudsen et al. 1995; Fig. 2). Such connections form an anatomical basis through which task-related information can influence saliency mapping in the OT (Winkowski and Knudsen 2008). It would therefore be of interest to study in a future project if this "top-down" pathway modulates SSA in the OT.

\section{Conclusions}

In recent years, the barn owl has emerged as an animal model for studying the neural mechanisms of saliency mapping and attention control. As evidence, two articles in this special issue are focused on stimulus selection in the OT of the barn owl. The overall findings in barn owls are strikingly similar to findings in other species, including primates. This similarity stresses the importance of a comparative approach to gain an evolutionary perspective on such basic elements of animal behavior. Recent findings show that SSA is pronounced specifically in areas related to gaze control and attention (reviewed above). We propose that specific adaptation is pronounced in the gaze control system as a part of its role in stimulus selection, giving rise to an attractive hypothesis that the neural mechanisms for highlighting a salient stimulus in space overlap with the mechanisms for highlighting a salient stimulus in time.

Acknowledgments This work was supported by grants from the Israel Science Foundation and the Institute for Psychobiology in Israel. The author thanks Amit Reches, Yael Zahar, and Shai Netser.

Open Access This article is distributed under the terms of the Creative Commons Attribution License which permits any use, distribution, and reproduction in any medium, provided the original author(s) and the source are credited.

\section{References}

Adolphs R (1993) Bilateral inhibition generates neuronal responses tuned to interaural level differences in the auditory brainstem of the barn owl. J Neurosci 13(9):3647-3668

Albeck Y, Konishi M (1995) Responses of neurons in the auditory pathway of the barn owl to partially correlated binaural signals. J Neurophysiol 74(4):1689-1700

Alho K (1995) Cerebral generators of mismatch negativity (MMN) and its magnetic counterpart (MMNm) elicited by sound changes. Ear Hear 16(1):38-51

Anderson LA, Christianson GB, Linden JF (2009) Stimulus-specific adaptation occurs in the auditory thalamus. J Neurosci 29(22): 7359-7363

Antunes FM, Nelken I, Covey E, Malmierca MS (2010) Stimulusspecific adaptation in the auditory thalamus of the anesthetized rat. PLoS One 5(11):e14071 
Bala AD, Takahashi TT (2000) Pupillary dilation response as an indicator of auditory discrimination in the barn owl. J Comp Physiol [A] 186(5):425-434

Barry RJ (2009) Habituation of the orienting reflex and the development of Preliminary Process Theory. Neurobiol Learn Memory 92(2):235-242

Bischof HJ, Watanabe S (1997) On the structure and function of the tectofugal visual pathway in laterally eyed birds. Eur J Morphol 35(4):246-254

Boehnke SE, Munoz DP (2008) On the importance of the transient visual response in the superior colliculus. Curr Opin Neurobiol 18(6):544-551

Bradley MM (2009) Natural selective attention: orienting and emotion. Psychophysiology 46(1):1-11

Brainard MS, Knudsen EI (1998) Sensitive periods for visual calibration of the auditory space map in the barn owl optic tectum. J Neurosci 18(10):3929-3942

Brosch M, Schreiner CE (1997) Time course of forward masking tuning curves in cat primary auditory cortex. J Neurophysiol 77(2):923943

Calford MB, Semple MN (1995) Monaural inhibition in cat auditory cortex. J Neurophysiol 73(5):1876-1891

Dong S, Clayton DF (2009) Habituation in songbirds. Neurobiol Learn Mem 92(2):183-188

Euston DR, Takahashi TT (2002) From spectrum to space: the contribution of level difference cues to spatial receptive fields in the barn owl inferior colliculus. J Neurosci 22(1):284-293

Eytan D, Brenner N, Marom S (2003) Selective adaptation in networks of cortical neurons. J Neurosci 23(28):9349-9356

Farley BJ, Quirk MC, Doherty JJ, Christian EP (2010) Stimulusspecific adaptation in auditory cortex is an NMDA-independent process distinct from the sensory novelty encoded by the mismatch negativity. J Neurosci 30(49):16475-16484

Fecteau JH, Munoz DP (2005) Correlates of capture of attention and inhibition of return across stages of visual processing. J Cogn Neurosci 17(11):1714-1727

Feldman DE, Knudsen EI (1997) An anatomical basis for visual calibration of the auditory space map in the barn owl's midbrain. J Neurosci 17(17):6820-6837

Furukawa S, Maki K, Kashino M, Riquimaroux H (2005) Dependency of the interaural phase difference sensitivities of inferior collicular neurons on a preceding tone and its implications in neural population coding. J Neurophysiol 93(6):3313-3326

Gaither NS, Stein BE (1979) Reptiles and mammals use similar sensory organizations in the midbrain. Science 205(4406):595-597

Glanzman DL (2009) Habituation in Aplysia: the Cheshire cat of neurobiology. Neurobiol Learn Memory 92(2):147-154

Gottlieb JP, Kusunoki M, Goldberg ME (1998) The representation of visual salience in monkey parietal cortex. Nature 391(6666):481484

Gutfreund Y, Knudsen EI (2006) Adaptation in the auditory space map of the barn owl. J Neurophysiol 17:17

Herrero L, Rodriguez F, Salas C, Torres B (1998) Tail and eye movements evoked by electrical microstimulation of the optic tectum in goldfish. Exp Brain Res 120(3):291-305

Horwitz GD, Newsome WT (1999) Separate signals for target selection and movement specification in the superior colliculus. Science 284(5417):1158-1161

Hyde PS, Knudsen EI (2000) Topographic projection from the optic tectum to the auditory space map in the inferior colliculus of the barn owl [In Process Citation]. J Comp Neurol 421(2):146-160

Ingham NJ, McAlpine D (2004) Spike-frequency adaptation in the inferior colliculus. J Neurophysiol 91(2):632-645

Itti L, Koch C (2000) A saliency-based search mechanism for overt and covert shifts of visual attention. Vision Res 40(10-12):1489-1506
Kane NM, Curry SH, Butler SR, Cummins BH (1993) Electrophysiological indicator of awakening from coma. Lancet 341(8846):688

Karten HJ, Hodos W, Nauta WJ, Revzin AM (1973) Neural connections of the "visual wulst" of the avian telencephalon. Experimental studies in the piegon (Columba livia) and owl (Speotyto cunicularia). J Comp Neurol 150(3):253-278

Katz Y, Heiss JE, Lampl I (2006) Cross-whisker adaptation of neurons in the rat barrel cortex. J Neurosci 26(51):13363-13372

Kayser C, Petkov CI, Lippert M, Logothetis NK (2005) Mechanisms for allocating auditory attention: an auditory saliency map. Curr Biol 15(21):1943-1947

King AJ, Hutchings ME (1987) Spatial response properties of acoustically responsive neurons in the superior colliculus of the ferret: a map of auditory space. J Neurophysiol 57(2):596-624

King AJ, Palmer AR (1985) Integration of visual and auditory information in bimodal neurones in the guinea-pig superior colliculus. Exp Brain Res 60(3):492-500

Knudsen EI (1982) Auditory and visual maps of space in the optic tectum of the owl. J Neurosci 2(9):1177-1194

Knudsen EI (1987) Neural derivation of sound source location in the barn owl. An example of a computational map. Ann N Y Acad Sci 510:33-38

Knudsen EI (2007) Fundamental components of attention. Annu Rev Neurosci 6:6

Knudsen EI (2011) Control from below: the role of a midbrain network in spatial attention. Eur J Neurosci 33(11):1961-1972

Knudsen EI, Knudsen PF (1983) Space-mapped auditory projections from the inferior colliculus to the optic tectum in the barn owl (Tyto alba). J Comp Neurol 218(2):187-196

Knudsen EI, Konishi M (1978) A neural map of auditory space in the owl. Science 200(4343):795-797

Knudsen EI, Cohen YE, Masino T (1995) Characterization of a forebrain gaze field in the archistriatum of the barn owl: microstimulation and anatomical connections. J Neurosci 15(7 Pt 2):5139-5151

Lai D, Brandt S, Luksch H, Wessel R (2011) Recurrent antitopographic inhibition mediates competitive stimulus selection in an attention network. J Neurophysiol 105(2):793-805

Lewald J, Dorrscheidt GJ (1998) Spatial-tuning properties of auditory neurons in the optic tectum of the pigeon. Brain Res 790(1-2): 339-342

Lovejoy LP, Krauzlis RJ (2009) Inactivation of primate superior colliculus impairs covert selection of signals for perceptual judgments. Nat Neurosci 13(2):261-266

Luksch H (2003) Cytoarchitecture of the avian optic tectum: neuronal substrate for cellular computation. Rev Neurosci 14(1-2):85-106

Malmierca MS, Cristaudo S, Perez-Gonzalez D, Covey E (2009) Stimulus-specific adaptation in the inferior colliculus of the anesthetized rat. J Neurosci 29(17):5483-5493

Marom S (2009) Neural timescales or lack thereof. Prog Neurobiol 90(1):16-28

Masino T, Knudsen EI (1992) Anatomical pathways from the optic tectum to the spinal cord subserving orienting movements in the barn owl. Exp Brain Res 92(2):194-208

McAlpine D, Jiang D, Shackleton TM, Palmer AR (2000) Responses of neurons in the inferior colliculus to dynamic interaural phase cues: evidence for a mechanism of binaural adaptation. J Neurophysiol 83(3):1356-1365

McHaffie JG, Stein BE (1982) Eye movements evoked by electrical stimulation in the superior colliculus of rats and hamsters. Brain Res 247(2):243-253

McPeek RM, Keller EL (2002) Saccade target selection in the superior colliculus during a visual search task. J Neurophysiol 88(4): 2019-2034

Middlebrooks JC, Green DM (1991) Sound localization by human listeners. Annu Rev Psychol 42:135-159 
Mill R, Coath M, Wennekers T, Denham SL (2011) A neurocomputational model of stimulus-specific adaptation to oddball and Markov sequences. PLoS Comput Biol 7(8):e1002117

Mize RR, Murphy EH (1976) Alterations in receptive field properties of superior colliculus cells produced by visual cortex ablation in infant and adult cats. J Comp Neurol 168(3):393-424

Moiseff A (1989) Bi-coordinate sound localization by the barn owl. J Comp Physiol A 164(5):637-644

Moiseff A, Konishi M (1983) Binaural characteristics of units in the owl's brainstem auditory pathway: precursors of restricted spatial receptive fields. J Neurosci 3(12):2553-2562

Muller JR, Metha AB, Krauskopf J, Lennie P (1999) Rapid adaptation in visual cortex to the structure of images. Science 285(5432): $1405-1408$

Muller JR, Philiastides MG, Newsome WT (2005) Microstimulation of the superior colliculus focuses attention without moving the eyes. Proc Natl Acad Sci USA 102(3):524-529

Mysore SP, Knudsen EI (2011) The role of a midbrain network in competitive stimulus selection. Curr Opin Neurobiol 21(4):653-660

Mysore SP, Asadollahi A, Knudsen EI (2010) Global inhibition and stimulus competition in the owl optic tectum. J Neurosci 30(5): 1727-1738

Mysore SP, Asadollahi A, Knudsen EI (2011) Signaling of the strongest stimulus in the owl optic tectum. J Neurosci 31(14):5186-5196

Naatanen R (1995) The mismatch negativity: a powerful tool for cognitive neuroscience. Ear Hear 16(1):6-18

Naatanen R, Tervaniemi M, Sussman E, Paavilainen P, Winkler I (2001) "Primitive intelligence" in the auditory cortex. Trends Neurosci 24(5):283-288

Nelken I (2004) Processing of complex stimuli and natural scenes in the auditory cortex. Curr Opin Neurobiol 14(4):474-480

Nelken I, Ulanovsky N (2007) Mismatch negativity and stimulusspecific adaptation in animal models. J Psychophysiol 21(3-4): 214-223

Netser S, Ohayon S, Gutfreund Y (2010) Multiple manifestations of microstimulation in the optic tectum: eye movements, pupil dilations, and sensory priming. J Neurophysiol 104(1):108-118

Netser S, Zahar Y, Gutfreund Y (2011) Stimulus specific adaptation: can it be a neural correlate of behavioral habituation? J Neurosci 31(49):17811-17820

Perez-Gonzalez D, Malmierca MS, Covey E (2005) Novelty detector neurons in the mammalian auditory midbrain. Eur $\mathrm{J}$ Neurosci 22(11):2879-2885

Pluta SR, Rowland BA, Stanford TR, Stein BE (2011) Alterations to multisensory and unisensory integration by stimulus competition. J Neurophysiol 106(6):3091-3101

Poganiatz I, Wagner H (2001) Sound-localization experiments with barn owls in virtual space: influence of broadband interaural level different on head-turning behavior. J Comp Physiol [A] 187(3):225-233

Poganiatz I, Nelken I, Wagner H (2001) Sound-localization experiments with barn owls in virtual space: influence of interaural time difference on head-turning behavior. J Assoc Res Otolaryngol 2(1): $1-21$

Posner MI (1980) Orienting of attention. Q J Exp Psychol 32(1):3-25

Posner MI (1981) Cognition and neural systems. Cognition 10(1-3): 261-266

Reches A, Gutfreund Y (2008) Stimulus-specific adaptations in the gaze control system of the barn owl. J Neurosci 28(6):1523-1533

Reches A, Gutfreund Y (2009) Auditory and multisensory responses in the tectofugal pathway of the barn owl. J Neurosci 29(30):96029613

Reches A, Netser S, Gutfreund Y (2010) Interactions between stimulusspecific adaptation and visual auditory integration in the forebrain of the barn owl. J Neurosci 30(20):6991-6998
Robinson DL, Petersen SE (1992) The pulvinar and visual salience. Trends Neurosci 15(4):127-132

Rodgers CK, Munoz DP, Scott SH, Pare M (2006) Discharge properties of monkey tectoreticular neurons. J Neurophysiol 95(6):35023511

Sams M, Paavilainen P, Alho K, Naatanen R (1985) Auditory frequency discrimination and event-related potentials. Electroencephalogr Clin Neurophysiol 62(6):437-448

Shimizu T, Karten HJ (1993) The avian visual system and the evolution of the neocortex. In: Zeigler HP, Bischof HJ (eds) Vision, brain, and behavior in birds. MIT, Cambridge, pp 103-114

Sokolov EN (1963) Higher nervous functions; the orienting reflex. Annu Rev Physiol 25:545-580

Sparks DL (1986) Translation of sensory signals into commands for control of saccadic eye movements: role of primate superior colliculus. Physiol Rev 66(1):118-171

Spitzer MW, Bala AD, Takahashi TT (2003) Auditory spatial discrimination by barn owls in simulated echoic conditions. J Acoust Soc Am 113(3):1631-1645

Stein BE, Meredith MA (1993) The Merging of the senses. Cognitive neuroscience series. MIT Press, Cambridge

Taaseh N, Yaron A, Nelken I (2011) Stimulus-specific adaptation and deviance detection in the rat auditory cortex. PLoS One 6(8):e23369

Takada M, Itoh K, Yasui Y, Sugimoto T, Mizuno N (1985) Topographical projections from the posterior thalamic regions to the striatum in the cat, with reference to possible tecto-thalamo-striatal connections. Exp Brain Res 60(2):385-396

Takahashi TT, Konishi M (1988) Projections of the cochlear nuclei and nucleus laminaris to the inferior colliculus of the barn owl. J Comp Neurol 274(2):190-211

Takahashi T, Moiseff A, Konishi M (1984) Time and intensity cues are processed independently in the auditory system of the owl. J Neurosci 4(7):1781-1786

Thompson RF (2009) Habituation: a history. Neurobiol Learn Memory 92(2):127-134

Thompson RF, Spencer WA (1966) Habituation: a model phenomenon for the study of neuronal substrates of behavior. Psychol Rev 73(1):16-43

Tiitinen H, May P, Reinikainen K, Naatanen R (1994) Attentive novelty detection in humans is governed by pre-attentive sensory memory. Nature 372(6501):90-92

Tsodyks MV, Markram H (1997) The neural code between neocortical pyramidal neurons depends on neurotransmitter release probability. Proc Natl Acad Sci USA 94(2):719-723

Ulanovsky N, Las L, Nelken I (2003) Processing of low-probability sounds by cortical neurons. Nat Neurosci 6(4):391-398

Ulanovsky N, Las L, Farkas D, Nelken I (2004) Multiple time scales of adaptation in auditory cortex neurons. J Neurosci 24(46):10440 10453

Valentinuzzi VS, Ferrari EA (1997) Habituation to sound during morning and night sessions in pigeons (Columba livia). Physiol Behav 62(6):1203-1209

Varela JA, Sen K, Gibson J, Fost J, Abbott LF, Nelson SB (1997) A quantitative description of short-term plasticity at excitatory synapses in layer $2 / 3$ of rat primary visual cortex. J Neurosci 17(20):7926-7940

von der Behrens W, Bauerle P, Kossl M, Gaese BH (2009) Correlating stimulus-specific adaptation of cortical neurons and local field potentials in the awake rat. J Neurosci 29(44):13837-13849

Wehr M, Zador AM (2005) Synaptic mechanisms of forward suppression in rat auditory cortex. Neuron 47(3):437-445

Weinberger NM, Oleson TD, Ashe JH (1975) Sensory system neural activity during habituation of the pupillary orienting reflex. Behav Biol 15(3):283-301 
Weisbard C, Graham PK (1971) Heart-rate change as a component of the orienting response in monkeys. J Comp Physiol Psychol 76(1):74-83

Winkler I, Denham SL, Nelken I (2009) Modeling the auditory scene: predictive regularity representations and perceptual objects. Trends Cogn Sci 13(12):532-540

Winkowski DE, Knudsen EI (2006) Top-down gain control of the auditory space map by gaze control circuitry in the barn owl. Nature 439(7074):336-339

Winkowski DE, Knudsen EI (2007) Top-down control of multimodal sensitivity in the barn owl optic tectum. J Neurosci 27(48):13279_ 13291
Winkowski DE, Knudsen EI (2008) Distinct mechanisms for top-down control of neural gain and sensitivity in the owl optic tectum. Neuron 60(4):698-708

Woods EJ, Frost BJ (1977) Adaptation and habituation characteristics of tectal neurons in the pigeon. Exp Brain Res 27(3-4):347-354

Zahar Y, Reches A, Gutfreund Y (2009) Multisensory enhancement in the optic tectum of the barn owl: spike count and spike timing. J Neurophysiol 101(5):2380-2394

Zimmer H (2006) Habituation of the orienting response as reflected by the skin conductance response and by endogenous event-related brain potentials. Int J Psychophysiol 60(1):44-58 